\title{
An asymptotic analysis of a self-similar solution for the double nonlinear reaction-diffusion system
}

\author{
M. Aripov ${ }^{1}$, Sh. Sadullaeva ${ }^{2}$ \\ ${ }^{1}$ National University of Uzbekistan named after M. Ulugbek, Tashkent, Uzbekistan \\ ${ }^{2}$ Tashkent University of information technology, Tashkent, Uzbekistan \\ mirsaidaripov@mail.ru,orif_sh@list.ru
}

PACS 02.30.Ik, 05.45.Yv

DOI 10.17586/2220-8054-2015-6-6-793-802

We study the solution for a system of reaction-diffusion equations with double nonlinearity in the presence of a source. A self-similar approach is used for the treatment of qualitative properties of a nonlinear reactiondiffusion system. It is shown that there exist some parameter values for which the effect of finite velocity of perturbation of distribution (FSPD), localization of solution, onside localization can occur. The problem for choosing the appropriate initial approximation for the iteration process used in numerical analysis is solved.

Keywords: reaction-diffusion system, double nonlinearity, qualitative properties.

Received: 1 November 2015

\section{Introduction}

Let us consider the Cauchy problem for the double nonlinear degenerate parabolic equation in the domain $Q=\left\{(t, x): t>0, x \in R^{N}\right\}$ :

$$
\begin{gathered}
A u \equiv-\frac{\partial u}{\partial t}+\nabla\left(u^{m-1}\left|\nabla u^{k}\right|^{p-2} \nabla u^{l}\right)-\operatorname{div}(v(t) u)+\gamma(t) u^{\beta}=0 \\
\left.u\right|_{t=0}=u_{0}(x) \geqslant 0, \quad x \in R^{N},
\end{gathered}
$$

where $\beta, p, m, l, k$ are the numerical parameters, $\nabla(\cdot)-\operatorname{grad}_{x}(\cdot), 0<\gamma(t) \in C(0, \infty)$.

Equation (1.1) is used for the modeling of various physical processes [1-7, 9-14], such as reaction-diffusion phenomena, heat conductivity, polytrophic filtration of gases and liquids in nonlinear media with source power of the form $\gamma(t) u^{\beta}$.

A specific property of this equation is its degeneration. In the domain where $u=0$ or $\nabla u=0$ Eq. (1.1) degenerates to a first order equation. Therefore, one needs to investigate the weak solution, since in this case, solutions of (1.1) may not exist in the classical sense [1].

The solution of Eq. (1.1) may exhibit interesting features, such as phenomenon of a finite speed of a propagation of distribution (FSPD), blow up [1] and localization of solution $[1,4,7,9,10,12-14,16,17]$. The FSPD was established first by Zeldovich-Kompaneets [1, 7], who constructed an exact self-similar solution for the following problem:

$$
\begin{aligned}
& \frac{\partial u}{\partial t}=\operatorname{div}\left(u^{m-1}|\nabla u|^{p-2} \nabla u\right), \\
& u(0, x)=P \delta(x) \geqslant 0, \quad x \in R^{N}
\end{aligned}
$$

where $\delta(x)$ is Dirac's delta function with the property of FSPD:

$$
u(t, x) \equiv 0,|x| \geqslant l(t), 0<l(t) \in C(0, \infty),
$$

and the constant $P>0$ is defined from condition $\int_{-\infty}^{\infty} u(t, x) d x=P$. 
Barenblatt (1952) and Pattle (1958) constructed solution (1.2) $(p=2)$ considering problem (1.3), (1.4) as the problem of a nonlinear diffusion and a problem of gas filtration. For the case $p=2$, and later for the case $p>2$ Martinson and Pavlov (see [4] and references) established the localization of solution for problem (1.3), (1.4) by constructing its exact solution.

The qualitative properties of these solutions for different classes and initial data, in the case $p=2$ or $m=1$ were investigated by many authors [4-23]. Global and blow up properties of the solution, as well as the numerical aspects for solving of the initial and boundary value problems for the equation (1.1) have also been studied by many authors [1-23].

In the context of applications in physics, it is reasonable to consider the weak solution obeying the condition:

$$
0 \leqslant u(t, x), u^{m-1}|\nabla u|^{p-2} \nabla u^{l} \in C(Q) .
$$

Definition 1. We shall call the solution with properties if there exists the continuous function $L(t)$ for $t>0$ such that $u(t, x) \equiv 0$ and for $|x| \geqslant L(t)$ the solution with a finite speed of propagation of disturbances.

The surface $|x|=L(t)$ is called a front of disturbance or a free boundary.

Definition 2. The solution of the Eq. (1.1) with properties $u(x, t) \equiv 0$ for $|x| \geqslant$ $L(t)<\infty, t>0$ is called the localized solution.

Definition 3. The solution, $u(x, t)$ is called a weak solution of the problem (1.1), (1.2) in $Q$, if $u(x, t) \geqslant 0$ almost everywhere for $(x, t) \in Q, 0 \leqslant u, u^{m-1}\left|\nabla u^{k}\right|^{p-2} \nabla u^{l} \in C(Q)$.

Then, the function $u(x, t)$ satisfies the integral identity:

$$
\left.\int_{0}^{t} \int_{\Omega}\left[-u \eta_{t}+u^{m-1}\left|\nabla u^{k}\right|^{p-2} \nabla u^{l} \nabla \eta\right) d x d t-\gamma(t) u^{\beta} \eta\right] d x d t+\int_{\Omega} u_{0}(x) \eta d x=0, \Omega \in R^{N}
$$

for any finite function $\eta(x, t) \in C_{0}^{1}(Q)$.

We note that the self-similar solution plays an important role in the study of qualitative properties for the solutions of the problem (1.1), (1.2). The self-similar analysis of the solution allows one to explore novel nonlinear effects in physics. In this work, we develop a method for constructing self-similar and approximately self-similar nonlinear reactiondiffusion equations and the method of nonlinear splitting (decomposition) and the method of standard equation [7] for their numerical solutions. Using these approaches, the numerical computations, visualization of solutions in animation form for one and two dimensional cases can be done. The numerical analysis of the solution presented is based on the use of Newton's linearization and Picard methods.

\section{Method of nonlinear splitting}

Our task is to solve the problem given by Eqs. (1.1), (1.2) using nonlinear splitting method.

We search for the solution of the equation (1.1) in the form:

$$
u(t, x)=\bar{u}(t) w(\tau, \xi), \xi=x-\int_{0}^{t} v(y) d y
$$


An asymptotic analysis of a self-similar solution...

where the function $\bar{u}(t)$ is the solution of the equation:

$$
\frac{d \bar{u}}{d t}=-\gamma(t) \bar{u}^{\beta}
$$

and $w(\tau, x)$ is the solution of the equation (1.1) without lower member.

Substituting Eq. (2.1) into (1.1), we obtain:

$$
\frac{\partial w}{\partial \tau}=\nabla\left(w^{m-1}\left|\nabla w^{k}\right|^{p-2} \nabla w^{l}\right)+\gamma(t) \bar{u}^{\beta-(k(p-2)+m+l-1)}\left(w+w^{\beta}\right),
$$

where $\tau(t)=\int[\bar{u}(t)]^{k(p-2)+m+l-2} d t$.

The main term in Eq. (2.2) has the self-similar solution of the kind:

$$
w(\tau, x)=f(\eta), \quad \eta=|\xi| /[\tau(t)]^{\frac{1}{p}} \quad .
$$

Substituting Eq.(2.3) into (2.2), we have the following approximately self-similar equation:

$$
\eta^{1-N} \frac{d}{d \eta}\left(\eta^{N-1} f^{m-1}\left|\frac{d f^{k}}{d \eta}\right|^{p-2} \frac{d f^{l}}{d \eta}\right)+\frac{\eta}{p} \frac{d f}{d \eta}+\gamma(t) \tau(t) \bar{u}^{\beta-(p+m+l-3)}\left(f+f^{\beta}\right)=0 .
$$

Let $\gamma(t)=(T+t)^{\sigma}$ where $\sigma$ is constant. Then,

$\tau(t)=\frac{1}{p_{1}}(T+t)^{p_{1}}$, if $p_{1}>0$,

$\tau(t)=\ln (T+t)$, if $p_{1}=0$,

with $p_{1}=1-(\sigma+1)(k(p-2)+m+l-2) /(\beta-1)$.

Therefore, Eq. (2.4) becomes self-similar:

$\eta^{1-N} \frac{d}{d \eta}\left(\eta^{N-1} f^{m-1}\left|\frac{d f^{k}}{d \eta}\right|^{p-2} \frac{d f^{l}}{d \eta}\right)+\frac{\eta}{p} \frac{d f}{d \eta}+\frac{\sigma+1}{\beta-1-(\sigma+1)(k(p-2)+m+l-2)}\left(f+f^{\beta}\right)=0$.

Different properties of solutions of Eq.(2.6) in particular cases, when $\sigma=0, p=2, m=k=l=1$, $\sigma=0, \quad m=k=l=1$ and for other particular numerical parameter values, have been studied by many authors (see, e.g. [1, 6-17]).

The case below,

$$
\beta=\beta_{c}=1+(\sigma+1)(k(p-2)+m+l-2)
$$

is a singular case. For the case $\sigma=0, k=l=1$, the properties of the positive solutions of the problem (1.1), (1.2) were studied by I. Combi [26].

\section{Global solvability and non-solvability of the Fujita-Samarskii type}

Let

$$
z_{+}(t, x)=\bar{u}(t) \bar{f}(\eta), \quad \bar{f}(\eta)=\left(a-b \eta^{p /(p-1)}\right)_{+}^{\frac{p-1}{q}}
$$

where

$$
q=k(p-2)+m+l-2, \quad b=[k(p-2)+m+l-2]\left(\frac{1}{p}\right)^{p /(p-1)} .
$$

Theorem 1. Let us consider the following conditions: $u_{0}(x) \leqslant z_{+}(0, x), x \in R^{N}, \beta>$ $k(p-2)+m+l-1+N / p$. Then, there exists a global solution $u(t, x)$ for the Cauchy problem (1.1), (1.2) for a small initial data with the following estimate:

$$
u(t, x) \leqslant z_{+}(t, x)
$$


in $Q$ and for the free boundary the estimate:

$$
\sum_{i=1}^{N}\left(x_{i}-\int_{0}^{t} v_{i}(t) d t\right)^{2} \leqslant(a / b)^{(p-1) / p} \tau^{1 / p}
$$

holds.

Corollary (generalization of the Fujita- Samarskii theorem).

Let

$$
\begin{gathered}
u_{0}(x) \leqslant z_{+}(0, x), x \in R^{N}, \quad \beta>(1+\sigma)(k(p-2)+m+l-2)+\frac{p}{N}, \\
a^{\frac{(p-1)(\beta-1)}{k(p-2)+m+l-2}}<\frac{N}{p}-\frac{1}{\beta-(1+\sigma)(k(p-2)+m+l-2)} .
\end{gathered}
$$

Then for solution of the problem (1.1), (1.2) in $Q$ the estimate:

$$
u(t, x) \leqslant z_{+}(t, x)
$$

holds true.

These results, with $\mathrm{l}=\mathrm{m}=1, \mathrm{p}=2$ in Eq.(1) have been proven in the Refs.[2, 3], and the case $\sigma=0, p=2, l=m=k=1$ has been treated by Samarskii A.A., Kurduomov S.P., Galaktionov V. A., Mikhaylov A. P., while the case $\sigma=0, l=m=k=1$ was studied by Galaktionov V.A. [1].

Proofs of these theorems and other propositions of the solution of problem (1.1), (1.2) are based on both the decomposition and comparison theorem method [1] as well as the use of function $\bar{f}(\eta)$ defined as the classical solution of equation:

$$
\eta^{1-N} \frac{d}{d \eta}\left(\eta^{N-1} f^{m-1}\left|\frac{d f^{k}}{d \eta}\right|^{p-2} \frac{d f^{l}}{d \eta}\right)+\frac{\eta}{p} \frac{d f}{d \eta}+\frac{N}{p} f=0
$$

on the domain $|\eta|<\left(\frac{a}{b}\right)^{\frac{p-1}{p}}$.

\section{Critical case}

The method of nonlinear splitting gives the explanation of the meaning of so-called "critical case":

$$
\gamma(t) \tau(t)[\bar{u}(t)]^{\beta-(p+m-2)}=N / p, \quad t>0 .
$$

For example, if $\gamma(t)=(T+t)^{\sigma}, \sigma>-1$, then the critical value of $\beta$ is given as:

$$
\beta=\beta_{*}=1+(\sigma+1)(k(p-2)+m+l-2)+p / N
$$

and the doubly critical case is

$$
\beta=\beta_{*}=1+(\sigma+1)(p / N) .
$$

This result for $m=k=l=1$ earlier was proved by Galaktıonov V. A., Vazquez J.L [1, 8, $10]$. 


\section{Mutual reaction-diffusion case}

We use self-similar analysis by constructing the exact solution the FSPD property and localization of weak solution from the class $0 \leqslant u, v, v^{m_{1}-1}\left|\nabla u^{k}\right|^{p-2} \nabla u^{l} \in C(Q)$, $u^{m_{2}-1}\left|\nabla v^{k}\right|^{p-2} \nabla v^{l} \in C(Q)$ solution of double nonlinear system in $Q$

$$
\begin{gathered}
\frac{\partial u}{\partial t}=\operatorname{div}\left(v^{m_{1}-1}\left|\nabla u^{k}\right|^{p-2} \nabla u^{l}\right)-\operatorname{div}(c(t) u)-\gamma_{1}(t) u, \\
\frac{\partial v}{\partial t}=\operatorname{div}\left(u^{m_{2}-1}\left|\nabla v^{k}\right|^{p-2} \nabla v^{l}\right)-\operatorname{div}(c(t) v)-\gamma_{2}(t) v \\
u(0, x)=u_{0}(x) \geqslant 0, \quad v(0, x)=v_{0}(x) \geqslant 0, x \in R^{N},
\end{gathered}
$$

where $\beta_{1}, \beta_{2}, p, m_{i}, k, l,(i=1,2)$ are given numerical parameters, $\nabla(\cdot)=\operatorname{grad}_{x}(\cdot)$. System is degenerating. Therefore we study a weak solution in above mentioned class. Using the transformation:

$$
\begin{gathered}
u(x, t)=(T+\tau(t))^{-\alpha_{1}} f(\xi), v(x, t)=(T+\tau(t))^{-\alpha_{2}} \psi(\xi), \\
\xi=|\eta| /[\tau(t)]^{1 / p}, \quad \eta=\int_{0}^{t} c(y) d y-x
\end{gathered}
$$

where

$$
\tau(t)=\int_{0}^{t} \exp \left(-n_{1} \int_{0}^{z} \gamma_{2}(y) d y\right) d z=\int_{0}^{t} \exp \left(-n_{2} \int_{0}^{z} \gamma_{2}(y) d y\right) d z
$$

for the functions $f(\xi), \psi(\xi)$ we have the degenerating self-similar system:

$$
\begin{aligned}
& \xi^{1-N} \frac{d}{d \xi}\left(\xi^{N-1} \psi^{m_{1}-1}\left|\frac{d f^{k}}{d \xi}\right|^{p-2} \frac{d f^{l}}{d \xi}\right)+\frac{\xi}{p} \frac{d f}{d \xi}+b_{1} f=0 \\
& \xi^{1-N} \frac{d}{d \xi}\left(\xi^{N-1} f^{m_{2}-1}\left|\frac{d \psi^{k}}{d \xi}\right|^{p-2} \frac{d \psi^{l}}{d \xi}\right)+\frac{\xi}{p} \frac{d \psi}{d \xi}+b_{2} \psi=0
\end{aligned}
$$

with

$$
\begin{aligned}
& \left.b_{1}=\alpha_{1} /\left[1-\alpha_{2}\left(m_{1}-1\right)-k(p-2)+l-1\right) \alpha_{1}\right], \\
& b_{2}=\alpha_{2} /\left[1-\left(m_{2}-1\right) \alpha_{1}-(k(p-2)+l-1) \alpha_{2}\right] .
\end{aligned}
$$

By constructing the following solution of Zeldovich-Barenblatt [1] type:

$$
\begin{aligned}
& u(x, t)=(T+\tau(t))^{-\alpha_{1}} \bar{f}(\xi), \\
& v(x, t)=(T+\tau(t))^{-\alpha_{2}} \bar{\psi}(\xi), \xi=|\eta| /[\tau(t)]^{1 / p},
\end{aligned}
$$

where

$$
\begin{gathered}
\bar{f}(\xi)=\left(a-\xi^{\gamma}\right)_{+}^{\gamma_{1}}, \quad \bar{\psi}(\xi)=\left(a-\xi^{\gamma}\right)_{+}^{\gamma_{2}}, \\
\gamma_{1}=\frac{\left.(p-1)\left(k(p-2)+l-m_{1}\right)\right)}{q}, \quad \gamma_{2}=\frac{\left.(p-1)\left(k(p-2)+l-m_{2}\right)\right)}{q}, \\
\gamma=p /(p-1), \quad q=k(p-2)+l-1-\left(m_{1}-1\right)\left(m_{2}-1\right), \quad \alpha_{1}=\frac{n_{2}}{n_{1}} \alpha_{2}, \\
\alpha_{2}=\frac{n_{2}}{n_{2}\left(p+n_{2} N\right)+\left(p-\left(n_{1}\left(m_{1}-1\right)\right) N\right.} .
\end{gathered}
$$


And using the self-similar analysis and comparison principle the following condition of a localization of a weak solution has proved the following:

$$
\begin{gathered}
\int_{0}^{t}\left(\exp \left(-n_{1} \int_{0}^{\eta} \gamma(y) d y\right) d \eta<\infty, \forall t>0, n_{1}=k(p-2)+l+m_{1}-2>0, \quad \int_{0}^{t} c(y) d y<\infty\right. \\
\int_{0}^{t}\left(\exp \left(-n_{2} \int_{0}^{\eta} \gamma(y) d y\right) d \eta<\infty, \forall t>0, \quad n_{2}=k(p-2)+l+m_{2}-2>0\right. \\
\tau(t)=\int_{0}^{t}\left(\exp \left(-n_{1} \int_{0}^{\eta} \gamma(y) d y\right) d \eta=\int_{0}^{t}\left(\exp \left(-n_{2} \int_{0}^{\eta} \gamma(y) d y\right) d \eta .\right.\right.
\end{gathered}
$$

Thus, the following weak solution with a localization property is found:

Theorem 2. We assume $\gamma_{1}>0, \gamma_{2}>0$. Then, the system (5.3) at $y \rightarrow \infty$ $\left(y=-\ln \left(a-\xi^{p /(p-1)}\right)\right)$ has an asymptotic:

$$
f(\xi)=A_{1} \bar{f}(\xi)(1+o(1)), \psi(\xi)=A_{2} \bar{\psi}(\xi)(1+o(1)),
$$

where the coefficients $A_{i}>0, \quad i=1,2$ are the solutions to the system of nonlinear algebraic equation:

$$
\begin{aligned}
& A_{1}^{k(p-2)+m+l-2} A_{2}^{m_{1}-1}=c_{1}, \quad c_{1}=\frac{1}{p\left(\gamma \gamma_{1}\right)^{p-1}}, \\
& A_{1}^{m_{2}-1} A_{2}^{k(p-2)+m+l-2}=c_{2}, \quad c_{2}=\frac{1}{p\left(\gamma \gamma_{2}\right)^{p-1}} .
\end{aligned}
$$

We also established the asymptotics of self-similar system in the fast diffusion case $\gamma_{1}<$ $0, \gamma_{2}<0$, and in the special case $q=0$. An asymptotic analysis of a self-similar system (5.3) also considered.

\section{Connection with a problem of the Kolmogorov-Fisher type biological population problem}

Fisher, Kolmogorov, Piskunov and Petrovski studied [27, 30, 33] wave type solutions of the problem of the biological population described by the following mathematical model:

$$
\begin{gathered}
A u \equiv-\frac{\partial u}{\partial t}+\nabla\left(D u^{m-1}\left|\nabla u^{k}\right|^{p-2} \nabla u^{l}\right)+k_{1} u\left(1-u^{\beta}\right)=0 \\
\left.u\right|_{t=0}=u_{0}(x) \geqslant 0, \quad x \in R^{N} .
\end{gathered}
$$

In the case when in (6.1) $k=m=l=1, p=2$. The numerical parameters $k, m, l \geqslant 1, p \geqslant 2$ for the velocity of wave type solution the estimate $c \geqslant 2(k D)^{1 / 2}$ was proved.

We notice that the following holds after the transformation:

$$
u(t, x)=e^{k_{1} t} w(\tau(t), x), \quad \tau(t)=\int_{0}^{t} e^{\left.k_{1}(p-2)+m+l-2\right) y} d y .
$$

Then, Eq. (6.1) can be rewritten as:

$$
\frac{\partial w}{\partial \tau}=\nabla\left(D w^{m-1}\left|\nabla w^{k}\right|^{p-2} \nabla w^{l}\right)-\gamma(\tau) u^{\beta}
$$


Thus, we reduced Eq.(6.1) into a form where, instead of the source, we have an absorption. Therefore, applying above method, one can use the self-similar analysis of the solutions to establish the novel nonlinear physical effects too [7].

One can show that for the problem (6.1)-(6.2) in the critical case $k(p-2)+l+m-2=0$ the speed $c$ of the wave distribution has the following estimate $\left.\frac{d(|x|)}{d t}=c \geqslant 2\left(D k_{1}\right)^{1 / p}\right) t^{(2 / p)-1}$ with depth of wave distribution for $t \sim \infty$.

$$
\left(|x(t)| \sim p\left(k_{1} D\right)^{1 / p} t^{2 / p}(1+o(1))\right.
$$

Since $u(x, t)=(T+t)^{-N / p} e^{k_{1} t-(\xi / p)^{p}}, \xi=|x(t)| /(T+t)^{1 / p}$ is a solution of a main part of the equation (6.2).

\section{Results of the numerical experiments and visualization of the solutions}

In the numerical solution for the above problem, the equation was approximated on a grid under the implicit circuit of variable directions (for a multidimensional case) in combination with the balance method. Iterative processes were constructed using Picard and Newton methods.

The results of numerical experiments show that the listed iterative methods are effective for the solution of nonlinear problems and leads to nonlinear effects if we will use as an initial approximation the solutions for self-similar equations constructed by nonlinear splitting and standard equation methods [7, 22, 23]. As was expected, for achievement of identical accuracy, the Newton method requires a smaller number of iterations than that of Picard's method or the special method, primarily due to the successful choice of an initial approximation. We observe that in each considered case, Newton's method has the best convergence due to good initial approximation.

The results of numerical experiments for the problem (1.1), (1.2) in the two-dimensional case are presented in the below table. For numerical solution of the problem, the method of the variable directions were applied.

The figures show that results of the numerical experiment gives the effect of a finite speed of a perturbation of solution, and localization of solution depending on the value of numerical parameters. The computational experiment were carried out for a slowly and a quick diffusion cases.

\section{Conclusion}

Using the self-similar approach, the localization of a solution for the equation with double nonlinearity was established. The influence of convective transfer to the process under consideration was studied. The significant role of the critical exponent was shown for the existing global and blow up solutions to the Cauchy problem for one equation with double nonlinearity with a convective transfer and a mutual (cross) system of parabolic equations. The appropriate initial approximation for the iteration process was suggested. The numerical computations, visualization of solutions in animation form were also discussed.

\section{Acknowledgments}

This work is supported by the grant of (Ref. Nr. A-5-44, 2015) of the Ministry of Higher Education of the Republic of Uzbekistan. 


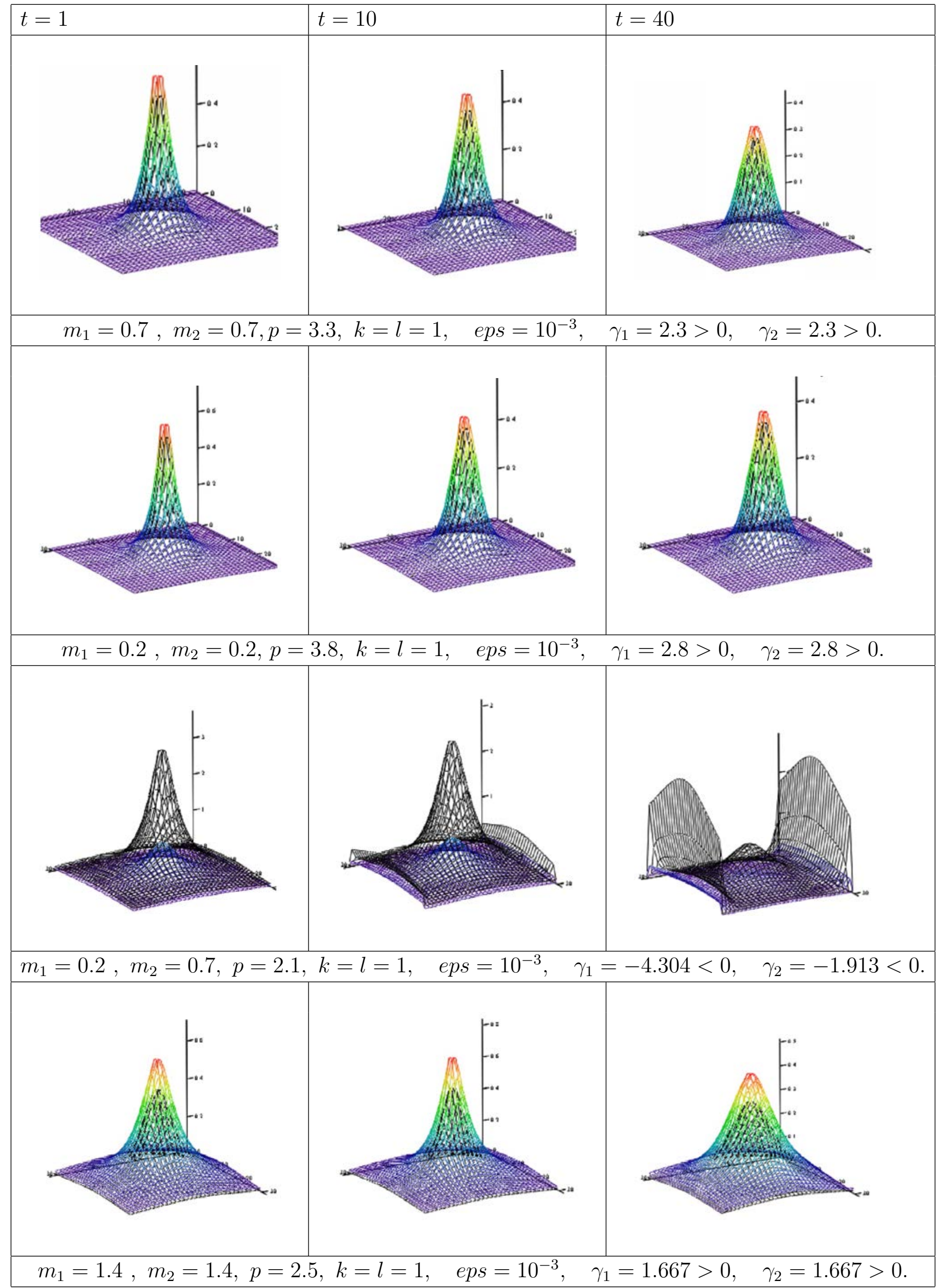




\begin{tabular}{|l|l|l|}
\hline$t=1$ & $t=10$ & $t=40$ \\
\hline & & \\
& \\
&
\end{tabular}

\section{References}

[1] Samarskii A.A., Galaktionov V.A., Kurduomov S.P., Mikhajlov A.P. Blowe-up in quasilinear parabolic equations. Berlin, 4, Walter de Grueter, 1995, p.535.

[2] Fujita H. On the blowing up of solutions to the Cauchy problem for $u_{t}=\Delta u+u^{1+\alpha}$, J. Fac. Sci. Univ. Tokyo Sect. I 13, 1966, P. 109-124.

[3] Hayakowa K. On nonexistence of global sol bal solutions of some semi linear parabolic differential equations, Proc. Japan Acad. Ser. A Math. Sci., 1973, 49, P. 503-505.

[4] Granik I.C. To localization of a temperature perturbation in a nonlinear medium with absorption. Journal of Comp. Math. Math. Phys. 1978, 18(3), P. 770-774.

[5] Deng K. and Levine H.A. The role of critical exponents in blow-up theorems: The sequel, J.Math. Anal. Appl. 2000, 243, P. 85-126.

[6] Levine H.A. The role of critical exponents in blow-up theorems, SIAM Rer., 1990, 32, P. 262-288.

[7] Aripov M. Standard Equation's Methods for Solutions to Nonlinear problems (Monograph), Tashkent, FAN, 1988, $137 \mathrm{p}$.

[8] Victor A. Galaktionov and J. L. Vazquez. The problem of blow-up in nonlinear parabolic equations. Discrete and continuous dynamical systems, April 2002, 8(2), P. 399-433.

[9] Tedeyev A.F. Conditions for the existence and nonexistence of a compact support in time of solutions of the Cauchy problem for quasilinear degenerate parabolic equations. Siberian Math. Jour., 2004, 45(1), P. 189-200.

[10] Vázquez J.L. The porous medium equation. Mathematical theory. Oxford Mathematical Monographs. The Clarendon Press, Oxford University Press, Oxford, 2007, 430 p.

[11] Chien-Hong Cho. On the computation of the numerical blow-up time. Japan Journal of Industrial and Applied Mathematics, 2013, 30(2), P. 331-349.

[12] Pan Zheng, Chunlai Mu. A complete upper estimate on the localization for the degenerate parabolic equation with nonlinear source. Mathematical methods in the Applied Sciences. Online publication date: 1-Jan-2014.

[13] M. Aripov, S. A. Sadullaeva. To properties of solutions to reaction-diffusion equation with double nonlinearity with distributed parameters. J. Siberian Federal University. Math. Phys., 2013, 6(2), P. 157-167.

[14] Aripov M. Asymptotes of the Solutions of the Non-Newton Polytrophic Filtration equation. ZAMM, 2000, $80(3)$, P. $767-768$.

[15] Martynenko A.V. and A.F. Tedeev. The Cauchy problem for a quasilinear parabolic equation with a source and inhomogeneous density, Comput. Math. Math. Phys., 2007, 47(2), P. 238-248.

[16] Martynenko A.V. and A. F. Tedeev, On the behavior of solutions to the Cauchy problem for a degenerate parabolic equation with inhomogeneous density and a source, Comput. Math. Math. Phys. 2008, 48(7), P. $1145-1160$.

[17] Afanas'eva N.V. and Tedeev A.F. Fujita type theorems for quasilinear parabolic equations with initial data slowly decaying to zero. Sbornik: Math., 2004, 195(1), P. 3-22. 
[18] Escobedo M., Levine H. A. Explosion et existence global pour un system habiliment couple deration direction diffusion. C. R. Acad. Sci. Ser.1, 1992, 314(10), P. 735-739.

[19] Chien-Hong Cho. On the computation of the numerical blow-up time. Japan Journal of Industrial and Applied Mathematics, 2013, 30(2), P. 331-349.

[20] Pan Zheng, Chunlai Mu, Iftikhar Ahmed. Cauchy problem for the non-Newtonian polytrophic filtration equation with a localized reaction. Applicable Analysis 1-16. Online publication date: 20-Feb-2014.

[21] Pan Zheng, Chunlai Mu. A complete upper estimate on the localization for the degenerate parabolic equation with nonlinear source. Mathematical methods in the Applied Sciences. Online publication date: 1-Jan-2014.

[22] Aripov M. Approximate Self-similar Approach for Solving of the Quasilinear Parabolic Equation. Experimentation, Modeling and Computation in Flow Turbulence and Combustion. Willey\&Sons, 1997, 2, P.9-26.

[23] Aripov M., Sadullaeva Sh. A. Properties of the solutions of one parabolic equation of non-divergent type. Proceedings of CAIM, 2003, 2, P. 130-136.

[24] Gilding B. H., Pelletier L. A. On a class of similarity of the porous media equation 2. J. Math. Anal. And Appl., 1977, 57, P. 522-538.

[25] Knerr B. F. The behavior of the support of solutions of the equation of nonlinear heat conduction with absorption in one dimension. Trans. of Amer. Math. Soc., 1979, 249, P. 409-424.

[26] Kombe I. Double nonlinear parabolic equations with singular lower order term, Nonlinear Analysis, 2004, 56, P. 185-199.

[27] Kolmogorov A.N., Petrovskii I.G., Piskunov N..S. Investigation of the equation of the diffusion connected to increase of quantity of substance and its application to one biological problem. Acta the Moscow State University, ser. Mathematics and mechanics, 1937, 1, P. 1-25.

[28] Kurduomov S.P., Kurkina E.S., Telkovskii. Blow up in two componential media. Mathematical Modeling, $1989, \mathbf{5}$.

[29] Aripov M. Self-Similar and Approximately Self-Similar Method to Solving of Problems of Nonlinear Unsteady a Filtration. Proceedings of the 1-st Turkish world mathematics symposium. Elazig/Turkey, 1999, P. 239-248.

[30] Marri Dj. Nonlinear diffusion equations in biology. M., Mir, 1983, 397 p.

[31] Dimova S.N., Kastchiev M.S., Koleva M.G., Vasileva D.P., Numerical analysis of the blow-up regimes of combustion of two-component nonlinear heat-conducting medium. JVM and MF, 1995, 35(3), P. 303319.

[32] Aripov M., Muhammadiev J. Asymptotic behavior of automodel solutions for one system of quasilinear equations of parabolic type. Buletin Stiintific-Universitatea din Pitesti, Seria Matematica si Informatica, $1999,3$.

[33] Holodnyok M., Klich A., Kubichek M., Marec M. Methods of analysis of dynamical models. Moscow, Mir, 1991, $365 \mathrm{p}$ 